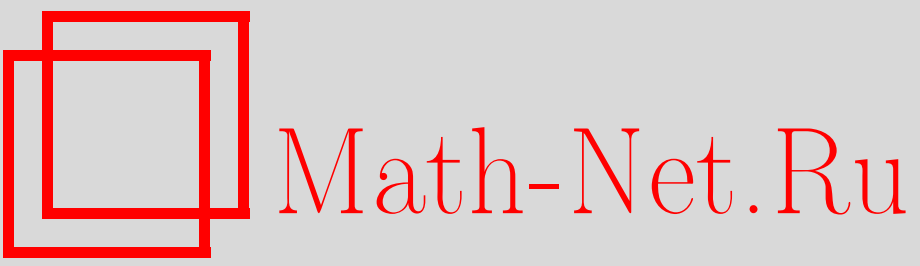

А. А. Туганбаев, Кусочно целостные кольца и несингулярно первичные идеалы, УМH, 1996, том 51, выпуск 4, 171-172

DOI: https://doi.org/10.4213/rm1009

Использование Общероссийского математического портала Math-Net.Ru подразумевает, что вы прочитали и согласны с пользовательским соглашением

http://www.mathnet.ru/rus/agreement

Параметры загрузки:

IP : 35.173 .137 .237

26 апреля 2023 г., 13:33:30 


\title{
КУСОЧНО ЦЕЛОСТНЫЕ КОЛЬЦА И НЕСИНГУЛЯРНО ПЕРВИЧНЫЕ ИДЕАЛЫ
}

\author{
А. А. ТуГАНБаЕв
}

Все кольца предполагаются ассоциативными и с единицей. Зафиксируем разложение $1=\sum_{i=1}^{n} e_{i}$ единицы кольца $A$ в сумму ортогональных идемпотентов $e_{i}$. Кольцо $A$ назьвается кусочно челостным (относительно $\left\{e_{1}, \ldots, e_{n}\right\}$ ), если оно удовлетворяет следующим трем эквивалентным [1] условиям: (1) для всех $e_{i}$ и $e_{k}$ каждый ненулевой гомоморфизм $e_{i} A \rightarrow e_{k} A$ является мономорфизмом; (2) для всех $e_{i}$ каждый ненулевой гомоморфизм $e_{i} A \rightarrow A_{A}$ является мономорфизмом; (3) $a b \neq 0$ для всех ненулевых элементов $a \in e_{j} A e_{i}, b \in e_{i} A e_{k}$ и для любых $e_{i}, e_{j}, e_{k}$. Поскольку условие (3) симметрично, то (1) и (2) можно заменить на их левосторонние аналоги. Модуль называется равномерныц, если любые его два ненулевых подмодуля имеют ненулевое пересечение. Модуль называется дистрибутивныцм, если решетка всех его подмодулей дистрибутивна. Идеал $B$ кольца $A$ будем называть кусочно целостныл, если $A / B$ - кусочно целостное (относительно естественных образов $\left\{e_{1}, \ldots, e_{n}\right\}$ в $A / B$ ) кольцо. Идеал $B$ кольца $A$ будем назьвать несингулярно первичным (вполне первичным), если $A / B$ - несингулярное справа первичное кольцо (область). Несингулярно первичный идеал кольца $A$, не содержащий других несингулярно первичных идеалов кольца $A$, называется минимальным несингулярно первичным идеалом.

ЗАмечание 1. $A$ - кусочно целостное кольцо $\Longleftrightarrow a b \neq 0$ для всех ненулевых $a \in A e_{i}$, $b \in e_{i} A$ и для каждого $e_{i}$.

ЗАмечАниЕ 2 . Если $F$ - собственный идеал кольца $A, h: A \rightarrow A / F$-естественный эпиморфизм, $e=e^{2} \in A \backslash F$, то: (1) $e F e$ - собственньй идеал кольца $e A e$ и существует естественный изоморфизм $h(e) h(A) h(e) \rightarrow e A e / e F e ;(2)$ хотя бы один ненулевой идемпотент $e_{i}$ не принадлежит $F$.

Замечания 1 и 2 проверяются непосредственно.

ЛЕмма 3. Если е - ненулевой идемпотент первичного кольиа $A$, то (еАе- область) $\Longleftrightarrow$ (любой ненулевой эндоморфизм правого идеала еА является мономорфизмом) $\Longleftrightarrow$ (любой ненулевой эндоморфизм каждого левого идеала Ае является мономорфизмом).

ДокАЗАтЕльство. Достаточно доказать первую эквивалентность. Импликация $=$ очевидна. Докажем $\Longrightarrow$. Так как $e A e$ можно естественно отождествить с $\operatorname{End}(e A)$, то достаточно привести к противоречию следующее утверждение: существуют такие ненулевые элементы $a=e a e \in e A e$ и $b=e b \in e A$, что $e a e b=0$. Тогда $(e a e)(e b A e)=0$. Так как $e A e-$ область и $e a e \neq 0$, то $e b A e=0$. Тогда $A e b A e A=0$. Поскольку $A$ первично, то либо $e b=0$, либо $e=0$, что невозможно.

Лемма 4. Пусть $M$ - ненулевой дистрибутивный правый идеал кольца А. Тогда:

(1) если $M=e A$, где $е=e^{2}$, то кольцо еАе дистрибутивно справа;

(2) если кольцо А первично, то модуль $М$ равномерен.

ДокаЗАтельСтво. (1) доказано в [2]. (2) Пусть $m, n \in M, a \in A$, причем $m A \cap n A=0$. Так как кольцо $A$ первично, то достаточно доказать, что $\operatorname{man}=0$. Правилом $f(n x)=\operatorname{manx}$ задается гомоморфизм $f \in \operatorname{Hom}(m A, n A)$. Так как модуль $M$ дистрибутивен и $m A \cap n A=0$, то $f \equiv 0[3]$. Поэтому $\operatorname{man}=0$.

ТеОРема 5. Пусть $F-$ первичный идеал кольца А. Тогда:

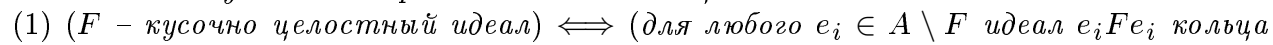
$e_{i} A e_{i}$ является вполне первичным );

(2) если все правые идеаль $\left(e_{i} A+F\right) / F$ кольца $A / F$ равномерны, то $(F-$ несингуляр-

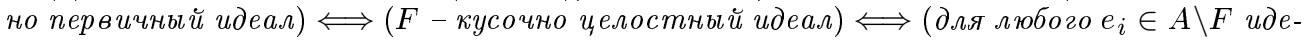
ал $e_{i} F_{i}$ кольиа е $e_{i} A e_{i}$ является вполне первичным $) \Longleftrightarrow\left(\right.$ хотя би для одного $e_{i} \in A \backslash F$ идеал е е $_{i}$ кольца е ${ }_{i} A e_{i}$ является вполне первичньм); 
(3) если все правые идеаль $e_{i} A$ дистрибутивны, то ( $F$ - несингулярно первичный идеал $) \Longleftrightarrow\left(F-\right.$ кусочно челостный идеал) $\Longleftrightarrow\left(\right.$ для любого $e_{i} \in A \backslash F$ идеал $e_{i} F e_{i}$ кольча $e_{i} A e_{i}$ является вполне первичным $) \Longleftrightarrow\left(e_{i} F e_{i}-\right.$ вполне первичный идеал кольца $e_{i} A e_{i}$ хотя бъ для одного $\left.e_{i} \in A \backslash F\right)$;

(4) если все правые идеалы $e_{i} A$ и все левые идеаль $A_{i}$ дистрибутивнь, то $(A / F-$ несингулярное справа кольцо $) \Longleftrightarrow(A / F-$ несингулярно слева).

ДоказАтельство. В силу замечания 2 можно считать, что $F=0$ и кольцо $A$ первичное. Докажем (1). Импликация $\Longrightarrow$ очевидна. Докажем $\Longleftarrow$. Допустим, что существуют $e_{i}$ и ненулевые $a=a e_{i} \in A e_{i}, b=e_{i} b \in e_{i} A$ такие, что $a e_{i} b=0$. Для любого $d \in A$ правилом $f_{d}\left(e_{i} x\right)=e_{i} d a e_{i} x$ задается эндоморфизм $f_{d}$ модуля $e_{i} A$. Так как $a e_{i} b=0$, то все $f_{d}$ не являются мономорфизмами. По лемме 3 все эндоморфизмы $f_{d}$ являются нулевыми. Поэтому $e_{i} A a e_{i} A=0$. Так как $A$ - первично, то либо $e_{i} A=0$, либо $a A=a e_{i} A=0$. Получено противоречие. По замечанию $1 A$ - кусочно целостное кольцо. Докажем (2). Первая эквивалентность вытекает из следующих фактов [1]: кусочно целостные кольца являются несингулярными; полуравномерные справа несингулярные справа кольца являются кусочно целостными. Вторая эквивалентность следует из (1). Докажем третью эквивалентность. Импликация $\Longrightarrow$ очевидна. Докажем $\Longleftarrow$. По (1) и по лемме 3 достаточно доказать, что для любого $j$ каждый эндоморфизм $f$ модуля $e_{j} A$, обладающий ненулевым ядром, тождественно равен нулю. Допустим, что $f\left(e_{j} A\right) \equiv N \neq 0$. Поскольку $A$ первично, то $N e_{i} A \neq 0$. Поэтому существует ненулевой гомоморфизм $h: e_{i} A \rightarrow N$. Поскольку $A$ первично, то $e_{i} A h\left(e_{i} A\right) \neq 0$. Поэтому существует ненулевой гомоморфизм $g: h\left(e_{i} A\right) \rightarrow e_{i} A$. Тогда $g h-$ ненулевой эндоморфизм модуля $e_{i} A$. Так как $e_{i} A$ - проективный модуль и $h\left(e_{i} A\right) \subseteq N \subseteq f\left(e_{j} A\right)$, то существует такой гомоморфизм $t: e_{i} A \rightarrow e_{j} A$, что $h=f t$. Тогда $t \neq 0$, поскольку $h \neq 0$. Так как $e_{j} A$ - равномерный модуль, то $M \equiv \operatorname{Ker}(f) \cap t\left(e_{i} A\right) \neq 0$. Поэтому $t^{-1}(M) \neq 0$, причем $(g h)\left(t^{-1}(M)\right)=(g f t)\left(t^{-1}(M)\right)=0$. По лемме $3 g h \equiv 0$ и получаем противоречие. (3) следует из (2) и леммы $4(2)$. (4) следует из (3).

ЗАмечАние 6 [3]. В дистрибутивном справа колце $A$ любые два не сравнимых по включению вполне первичных идеала равны в сумме $A$.

Следствие 7. Пусть все правъе идеаль $e_{i}$ А кольца А дистрибутивны. Тогда:

(1) если $F$ и $G$ - несингулярно первичные идеаль кольца $A$, то либо $F+G=A$, либо $F$ и $G$ сравнимы по включению;

(2) каждый собственный несингулярно первичный идеал F кольца $A$ содержит ровно один минимальный несингулярно первичный идеал.

ДоказАтельство. Докажем (1). Допустим, что $F+G \neq A$. Найдется $e_{i} \equiv e \in A \backslash(F+G)$. По лемме 4(1) кольцо $e A e$ дистрибутивное справа, а по (1) $e F e, e G e$ - его вполне первичные идеалы, причем $e A e \neq e F e+e G e$. В силу замечания 6 можно без ограничения общности считать, что $e F e \subseteq e G e$. Так как $G$ - первичньй идеал, причем $e \in A \backslash G$ и $e F e \subseteq G$, то $F \subseteq G$. Докажем (2). В силу (1) и леммы Цорна достаточно доказать, что если $G=\bigcap_{j \in J} G_{j}-$ пересечение убывающей цепи лежащих в $F$ несингулярно первичных идеалов $G_{j}$, то $G$ - несингулярно первичный идеал. Так как все идеалы $G_{j}$ первичны, то $G$ - первичньй идеал. Пусть $e_{i} \equiv e \in A \backslash F$. По теореме 5(3) все $e G_{j} e-$ вполне первичные идеалы кольца $e A e$. Тогда $e G e=\bigcap_{j \in J} e G_{j} e-$ вполне первичный идеал. По теореме $5(3) G$ - несингулярно первичный идеал.

\section{СПИСОК ЛИТЕРАТУРЫ}

[1] Gordon R., Small L. W. // J. Algebra. 1972. V. 23. P. 553-564. [2] Туганбаев A. A. // УMH. 1996. T. 51. №3. C. 201-202. [3] Stephenson W. // Proc. London Math. Soc. 1974. V. 28. № 2. P. 291-310. 